\title{
Beyond Alterity: los fantasmas del indigenismo bajo escrutinio
}

\section{Beyond Alterity: The ghosts of indigenism under scrutiny}

\author{
Luis Acatzin Arenas Fernández \\ doi) doi.org/10.29043/liminar.v20i2.923
}

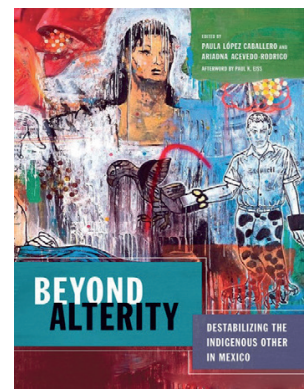

Resumen: Reseña de Beyond Alterity. Destabilizing the Indigenous Other in Mexico, editado por Ariadna Acevedo Rodrigo y Paula López Caballero. Tucson: Arizona University Press, 2018.

Abstract: Review of Beyond Alterity. Destabilizing the Indigenous Other in Mexico, edited by Ariadna Acevedo Rodrigo and Paula López Caballero. Tucson: Arizona University Press, 2018.

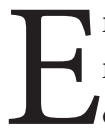
n abril de 2016, las diputadas que integraban la Comisión de Derechos Humanos y Grupos Vulnerables del Congreso de Guanajuato se reunieron con representantes de diferentes grupos indígenas que pedían oportunidades de trabajo y educación. Ante estas demandas, la diputada priista Luz Elena Govea respondió: "se los digo con todo respeto, cuando ustedes piden trabajo es necesario que manifestemos cuáles son nuestras aptitudes [...] no me imagino a las mujeres indígenas en una fábrica, haciendo el aseo de un edificio, detrás de un escritorio. Me las imagino en el campo, en sus casas haciendo artesanías, haciendo el trabajo de sus comunidades indígenas" (Espinoza, 2016, cursivas añadidas).

Posteriormente, la diputada intentó interpelar a sus interlocutores e interlocutoras como el cuerpo material que da vida a la comunidad imaginada (Anderson, 1993) que llamamos México: "a eso dirijamos la visión del trabajo que ustedes están solicitando, porque si ustedes deciden abandonar su tierra y sus tradiciones, el pueblo mexicano nos quedamos sin nuestras raíces. Piensen en eso cuando quieran ver un futuro" (Espinoza, 2016, cursivas añadidas). Para la política priista era importante hacer saber a nuestros indígenas de la nación

Recibido: 10 de agosto de 2021 Aprobación: 18 de agosto de 2021 Publicación: 28 de enero de 2022
* Centro de Investigaciones y Estudios Superiores en Antropología Social-Unidad Sureste, México acatzin.arenas.fdez@gmail.com (i) 0000-0001-5975-1499 
(López, 2017) la relevancia de la labor que realizan simplemente siendo quienes son: "la dignidad con la que ustedes visten, cargan ese rebozo o calzan esos zapatos. Es parte de lo que nosotros les imitamos". Con su argumento de cierre, la alteridad dejó de ser una característica propia de toda sociedad y se convirtió en un valor en sí mismo: "creo que algunos nos ganamos la vida detrás de un escritorio, otros labrando la tierra, otros cortando los nopalitos. Entiendo que el trabajo es duro, sin embargo, analicemos y pensemos en ese futuro como pueblos indígenas, ustedes no pueden ser sustituidos por nadie" (Espinoza, 2016).

Si bien podemos reconocer que los comentarios de la diputada son desafortunados, tal vez la indignación que generaron no fue resultado de las ideas en sí mismas, sino de la manera tan directa y despreocupada en que fueron expuestas y, claro, por el contexto en que se expresaron. Esto es, ¿acaso no es algo generalizado asumir que se sabe bien cuál es el lugar de los indígenas en México?, ¿no es común imaginar al indígena como una persona que tiene tradiciones auténticas, como un creyente que realmente cree, como artista que toma de la naturaleza inspiración y sustento? En palabras más directas, ¿el indígena no suele ser representado dando por hecho su alteridad como una característica innata? El caso de la diputada es llamativo en la medida en que volvió evidente e intolerable, al menos por un momento, las certezas vigentes en torno a lo que se piensa que es la persona indígena y el lugar que le corresponde de acuerdo con sus diferencias, es decir, la alteridad que se asume es su sello esencial. Pero, ¿qué sucede cuando, a diferencia del ejemplo anterior, estas ideas pasan desapercibidas como parte de la vida cotidiana?, ¿cuál es el proceso mediante el cual se han naturalizado?

\section{II}

Beyond Alterity. Destabilizing the Indigenous Other in Mexico, es una compilación de trabajos que reviven los fantasmas del indigenismo. Que quien se acerque al texto no dude de esta empresa, pues su intención no es replicar las preguntas del proyecto académico y político que se cuestiona qué es el pueblo indígena y qué hacemos con él. Mas bien, en las dos secciones que integran el libro, Land and Government (capítulos 1 al 6) y Science (capítulos 7 al 11), la historia es interrogada para profundizar en los procesos que han definido qué y quién es indígena. Esta obra, producto de un grupo de trabajo auspiciado por la beca para seminarios Mellon-Latin American Studies Association (LASA), inicia con una pregunta propia que sirve como introducción: Why Beyond Alterity? Desde ahí, las editoras Paula López Caballero ${ }^{1}$ y Ariadna Acevedo Rodrigo ${ }^{2}$ se aproximan al espinoso tema del sujeto indígena y su vinculación con la alteridad. La ruta que toman es la del análisis histórico de ambas nociones, tanto de su construcción como de las formas en que han sido experimentadas por sujetos concretos. Para ello,

\footnotetext{
${ }^{1}$ López Caballero es licenciada en historia por la Universidad Nacional Autónoma de México y recibió el grado de maestra y doctora en Antropología Social y Etnología por la École des Hautes Études en Sciences Sociales de París. Es autora de Indígenas de la Nación, libro publicado por el Fondo de Cultura Económica en 2017. Actualmente es investigadora del Centro de Investigaciones Interdisciplinarias en Ciencias y Humanidades de la Universidad Nacional Autónoma de México (CIICHUNAM). Sus intereses de investigación están dirigidos hacia los procesos históricos que han definido qué y quién es indígena.

${ }^{2}$ Acevedo Rodrigo es licenciada en Ciencias Política y Sociología por la Universidad Complutense de Madrid y se tituló como maestra y doctora en Historia en la Universidad de Warwick, en Reino Unido. Es coeditora del libro Ciudadanos inesperados. Espacios de formación de la ciudadanía de ayer y hoy (Acevedo y López, 2012), el cual lleva el sello de la editorial de El Colegio de México. Acevedo Rodrigo es miembro del Sistema Nacional de Investigadores (SIN) y está adscrita al Centro de Investigación y de Estudios Avanzados (Cinvestav). La relación entre educación y política durante los siglos XIX y XX es su principal interés de investigación.
} 
cuestionan las certezas formuladas acerca del tema sin emitir juicios de valor para descartar a los falsos indígenas, ni con la intención de señalar la verdadera alteridad. Mas bien, evitando toda pretensión de mostrar al público lector cuál es el camino que llevará al cierre de la discusión, plantean como punto de partida problematizar la noción de indígena. Al darle la vuelta a esta categoría, convirtiéndola en un objeto de estudio que debe ser explicado y no en un argumento explicativo, dan un paso fuera del atolladero que el juego de las definiciones y de las antinomias (indígena/no-indígena) ha generado.

Esta propuesta implica romper los límites en los que el indigenismo se ha encerrado, los cuales, a lo largo de la historia de las Ciencias Sociales y las Humanidades en México, han ocupado a mentes brillantes en discusiones, podríamos decir, bizantinas. Se trata, por un lado, de la inagotable tarea de distinguir entre indígenas y no-indígenas. A pesar de los muchos intentos por discriminar ambos grupos de manera exacta (científica) apoyándose en rasgos culturales, zonas geográficas, lengua, vestimenta, organización política, propiedad comunal, historia, la autoidentificación y hasta la genética, o con diferentes y contradictorias combinaciones de estos elementos; como bien lo muestran los trabajos que integran este libro, distinguir grupos/individuos guiándose por estos criterios no lleva a conclusiones claras ni coherentes. Aún más, el mismo intento de definición y diferenciación termina por inventar aquello que se intenta conocer, de tal manera que la empresa indigenista es como la serpiente que se muerde la cola: autorreferencial. Cada vez que parece que se logra fijar la identidad indígena, esta se vuelve escurridiza y las categorías tan solo permanecen como ortodoxia.

Por otro lado, el indigenismo suele caer en la trampa del exotismo al dotar de personalidad, arbitrio y cohesión a abstracciones que son problemáticas, entre ellas, las siempre ambiguas nociones de pueblo, comunidad y grupo étnico. De acuerdo con López Caballero y Acevedo Rodrigo, estos conceptos, así como el de indígena, deben de ser abordados como campos de lucha, resistencia y negociación en constante reformulación, pues en su seno existen antagonismos que los hacen cambiantes, es decir, históricos. Por lo tanto, sugieren, es necesario entenderlos en relación con los contextos políticos que los moldean, tanto el local como el más amplio (Estado-nación), relacionando de esta forma escalas de orden político. Esta manera de problematizar la categoría de indígena y de mirar con ojos críticos la alteridad no tiene como objetivo, señalan las editoras, menospreciar las luchas particulares que se amparan en estas ideas. Mas bien, tomando como referencia dichas luchas, se busca dar cuenta de las experiencias que van más allá de la dureza de los conceptos. Experiencia es, entonces, otro de los conceptos clave que atraviesa los estudios presentados en las 312 páginas que integran la obra.

Si bien el concepto de experiencia puede llegar a ser tan ambiguo, romántico y abarcador como lo son cultura e identidad, como habrían de decir Victor Turner y Edward Bruner (1986), cuenta con la virtud de estar vinculado con la fluidez de la vida y, por lo tanto, resalta el cambio y la diversidad. Mientras que buena parte de la bibliografía concentrada en lo indígena moviliza como herramientas teóricas y políticas las diferencias identitarias y las permanencias culturales, en Beyond Alterity el análisis se inspira en lo que las editoras llaman una "antropología política de la identificación". Ante ello, es difícil no recordar las reflexiones de Stuart Hall quien, al preguntarse quién necesita identidad, interroga este concepto como reflejo de perspectivas esencialistas y primordialistas y, al no encontrar una forma más específica de abordar las subjetividades, llamó identificación a los procesos temporales de interpelación ideológica que dan forma a los sujetos (Hall, 2011). La característica de las identificaciones es que se construyen dentro de campos de lucha política e ideológica, cruzando diferentes flujos discursivos y de prácticas que hacen de la subjetividad algo cambiante y múltiple. 
Las y los investigadores que participan en la obra aquí reseñada llaman la atención acerca de la relevancia que tiene el Estado en el uso de la identificación indígena, la cual es parte del lenguaje oficial para dirimir conflictos y organizar la relación con las instituciones, y característica de legitimación. El lenguaje cultural movilizado por el Estado, por un lado, puede llegar a ser extremadamente efectivo en la medida en que es naturalizado y se adhiere a las estructuras profundas que preceden y moldean la experiencia de los individuos. Es decir, producen subjetividades y establecen pautas de interacción. En ese sentido, la diada alteridad/indígena, se sugiere en Beyond Alterity, puede ser entendida como un efecto del Estado.

Por otro, como lo ha hecho notar William Roseberry (2002), todo proyecto hegemónico impulsado por el Estado, instancia que requiere de la acción conjunta de un marco discursivo común (lenguaje contencioso) y del uso de la fuerza (coerción), nunca es un programa acabado. Mas bien, siempre es un proyecto en proceso de construcción, de modo que una de sus características principales es la fragilidad. Esto se debe a que, y aquí también recupero a Raymond Williams (2000), no hay hegemonía que pueda agotar la creatividad y la energía humanas. En otras palabras, la experiencia siempre excede a las categorías y a los proyectos de dominación, los cuales requieren de constante renovación para seguirles el paso. Tal vez este sea uno de los puntos de análisis más importantes de esta compilación de investigaciones. Como lo aclaran las editoras, en ocasiones la identificación indígena, así como algunos proyectos del Estado que el propio Estado no tiene el interés o la capacidad de desplegar de manera amplia, son retomados por los sujetos, quienes se los apropian, los acoplan a sus necesidades y los usan a su favor, llegando, en ocasiones, a invertir las jerarquías dominantes.

En ese sentido, en Happy Together? "Indians", Liberalism, and Schools in the Oaxaca and Puebla Sierras, 1876-1911, cuarto capítulo de la obra reseñada, Acevedo Rodrigo nos invita a pensar el proyecto liberal de educación del siglo XIX, no como empresas marcadas por el fracaso, ni simplemente como imposición de un lenguaje o la homogeneización de una manera de pensar. Como muestra en su investigación, ciertos pueblos y aldeas invirtieron tiempo, energía y dinero para atraer y mantener escuelas que permitieran educar a su población. Si bien la escuela reprodujo relaciones jerarquizadas de poder, también brindó los medios para cuestionarlas. Este dato simultáneamente es muestra de la capacidad que las poblaciones indígenas han tenido para estar conectadas con el exterior, con lo cual se rompe la noción de comunidades encerradas en sus núcleos. De acuerdo con Peter Guardino, dichas comunidades estaban interconectadas con ámbitos más amplios, lo cual demuestra en su investigación Connected Communities: Villagers and Wider Social Systems in the Late Colonial and Early National Periods (cap. 2), al identificar la apropiación, por parte de estas comunidades, del lenguaje legal y político de la época tardía colonial, y del vocabulario característico de los discursos políticos modernos (ciudadanía, soberanía popular y autonomía municipal).

Asimismo, Michael T. Ducey, en Indigenous Communities, Political Transformations, and Mexico's War of Independence in the Gulf Coast Region (cap. 3), resalta que las comunidades indígenas contaban con extensas conexiones políticas. Además de buscar patrocinios en otras zonas, entendían que sus intereses inmediatos, entre ellos la autonomía municipal, estaban condicionados por sucesos más amplios que ocurrían en otros lugares. Sin embargo, la capacidad de negociar no era trabajo ni cualidad de la comunidad. De acuerdo con Elsie Rockwell, solo ciertas personas lograron apropiarse de la alfabetización en español, lo cual significa que, además de entender el idioma castellano, aprendieron el lenguaje del ambiente político y administrativo. Esto, como señala Rockwell en Todos tenemos la crisma de dios: Engaging Spanish Literacy in a Tlaxcalan Pueblo (cap. 5), les permitió operar como traductores de ese sistema y, ya sea para beneficio propio o de sus comunidades, modificar documentos escritos a partir de prácticas de la memoria. 
Otro tema relevante abordado en el libro es la fuerza que tiene en la imaginación de los no-indios la imagen fantasmal de aquello que Francisco de la Peña (2002) ha llamado un otro otro. Esto es, no la persona indígena real de carne y hueso, sino lo que los mestizos fantasean ver en ellos. En su análisis Displacement, Development, and the Creation of a Modern Indigena in the Papaloapan, 1940s-1970s (cap. 9), en el que se aborda el proyecto impulsado por el Instituto Nacional Indigenista (INI) en el Papaloapan, Diana Lynn Schwartz, concluye que la pretensión de modernizar al indígena sin permitir que perdiera su alteridad fue un fracaso, no por falta de voluntad, sino "porque los indígenas, tanto como individuos como en comunidades, eran ilusorios" (p. 237).

A pesar de que nociones como raza y biología han perdido legitimidad desde el siglo XX, como señala Vivette García Deister en In Sickness and in Myth: Genetic Avatars of Indigenous Alterity and the Mexican Nation (cap. 11), la insistencia en encontrar la raíz de una alteridad indígena imaginada ha llegado a moldear, incluso, la manera en que se estudia el mapa genómico del individuo mexicano. Si bien estos estudios intentan conocer más acerca de ciertas enfermedades, terminan creando una doble alteridad del indígena: una patológica y otra mítica (p. 266). En el mismo tenor, Laura Cházaro muestra cómo, en el siglo XIX, con la finalidad de encontrar una lógica en la variabilidad de las razas humanas, se combinó la exactitud de herramientas y mediciones científicas, con las ambiguas ideas antropológicas acerca del elemento racial. Con ello, como se muestra en From Anatomical Collection to National Museum, circa 1895: How Skulls and Female Pelvises Began to Speak the Language of Mexican National History (cap. 7), huesos y esqueletos se convirtieron en pruebas materiales de la alteridad.

Puesto que la antropología tuvo un rol protagónico en la construcción de esta representación imaginaria de la alteridad indígena, Paula López Caballero en Anthropological Debates Around the Indigenous Subject and Alterity, 1940-1948 (cap. 8) y José Luis Escalona Victoria en Encapsulated History: Evon Vogt and the Anthropological Making of the Maya (cap. 10), profundizan en el proceso de institucionalización de la disciplina. López Caballero, al reflexionar desde la noción de "espacio de polémica", analiza la intersección entre políticas indigenistas, antropología y la sociogénesis del concepto indígena, para mostrar que las ideas vigentes en torno a lo indígena surgieron de una combinación entre las características del estudio de la raza (continuidad y herencia), y la noción cultural de pertenencia a una comunidad. Escalona Victoria, por su parte, recupera la trayectoria en el sureste mexicano del antropólogo estadounidense Evon Vogt, quien llegó a esta zona fascinado por las historias de la antigua civilización maya, la cual ya era objeto de estudio de un amplio círculo de investigadores. Vogt creyó haber encontrado una lógica cultural regional preservada desde la época prehispánica, la cual estudió sin considerar la influencia del tiempo ni de los procesos políticos, económicos y culturales que la moldearon. Es por ello que, de acuerdo con Escalona, Vogt encapsuló la historia de los mayas y con ello contribuyó tanto a su producción como a su institucionalización.

La imaginada alteridad indígena también suele estar asociada a la noción de comunalidad, la cual se asume que tiene un origen prehispánico. En el primer capítulo del libro, titulado The Practices of Communal Landholding: Indian Pueblo Property Relations in Colonial Mexico, Emilio Kourí rompe con la imagen estereotipada de la propiedad comunal y nos dice que, durante el siglo XVIII, los indígenas tenían mayor libertad de lo que pensamos para heredar, comprar y vender tierras. Si bien la constitución 
de ejidos en el siglo XX puso límites a esta libertad, y a pesar de la idea dominante de que estos son una continuación de la cosmovisión de los pueblos indígenas, de acuerdo con Gabriela Torres-Mazuera a cargo del capítulo 6 Communal and Indigenous Landholding in Contemporary Yucatan: Tracing the Changing Property Relations in the Postrevolutionary Ejido, la gente siempre busca formas de brincarlos. En Yucatán, algunos pueblos mayas convirtieron las parcelas ejidales en colectivas, esto como una estrategia para sostener una relación más flexible e individual con la propiedad de la tierra.

Puesto que la alteridad indígena no es más que el salvaje en el espejo (Bartra, 1998) en el que Occidente se ha reflejado y construido, en el epílogo Beyond Alterity, Beyond Occidentalism: "Indigenous Other" and "Western Self" in Mexico, Paul K. Eiss hace un llamado a cuestionar la noción de occidentalismo a la par de la de alteridad. Al recuperar la obra de Guillermo Bonfil, Eiss sugiere que aquel reconocido antropólogo mexicano estaba proponiendo revertir las posiciones ocupadas por el México profundo y el México imaginario, de modo que lo indígena dejara de ser "lo otro" y el mexicano se pudiera reflejar en ello como un self. Sin embargo, se pregunta si este cambio de polaridades que continúa sustentado en esencialismos no termina tan solo reproduciendo el mismo problema. Tal vez, para lograr ir más allá de la alteridad asignada a los indígenas (nativos, originarios, tribus), y para derrumbar la presunta universalidad del occidental (colono, blanco), es necesario, como ha sugerido Mahmood Mamdani (2020) en el caso africano, cortar de tajo la construcción de identidades políticas que surgen de este juego de oposiciones.

En sintonía con estas ideas, los trabajos reunidos en Beyond Alterity muestran que el nosotros ha sido pieza clave para producir las diferencias asignadas a ellos, los otros, los indígenas. De igual forma, dejan claro a sus lectores que aquellos antropólogos e historiadores que intentaron resolver estos temas terminaron colaborando en la construcción del problema que buscaban solucionar. A decir de Slavoj Žižek (Strom Života, 2011), el trabajo del intelectual no puede ser el del experto que brinda soluciones. En ocasiones, el problema es cómo percibimos un problema, por lo que detectarlo y generar nuevas preguntas que brinden una perspectiva distinta es un aporte valioso. Beyond Alterity hace precisamente eso, no busca dar recetas mágicas, sino hacer de un problema teórico y práctico un objeto de estudio en sí mismo.

\section{Referencias}

Acevedo Rodrigo, A., y López Caballero, P. (2012). Ciudadanos inesperados. Espacios de formación de la ciudadanía de ayer hoy. Centro de Investigación y de Estudios Avanzados; El Colegio de México.

Anderson, B. (1993). Comunidades imaginadas. Reflexiones sobre el origen y la difusión del nacionalismo. Fondo de Cultura Económica.

Bartra, R. (1998). El salvaje en el espejo. Era.

De la Peña, F. (2002). Los hijos del sexto sol. Instituto Nacional de Antropología e Historia.

Espinoza, V. (2016 6 de abril). Diputada aconseja a indígenas: "no dejen de vender sus nopalitos y artesanías". Proceso. https://www.proceso.com.mx/nacional/estados/2016/4/6/diputada-aconseja-indigenas-no-dejende-vender-sus-nopalitos-artesanias-162153.html

Hall, S. (2011). Introducción: ¿Quién necesita Identidad? En S. Hall y P. Du Gay (coords.), Cuestiones de identidad cultural (pp. 13-39). Amorrortu.

López, P. (2017). Indígenas de la nación: etnografía histórica de la alteridad en México (Milpa Alta S. XVII-XXI). Fondo de Cultura Económica. 
Mamdani, M. (2020). Neither settler nor native. The making and unmaking of permanent minorities. Belknap Press.

Roseberry, W. (2002). Hegemonía y el lenguaje contencioso. En J. Gilbert y D. Nugent (coords.), Aspectos cotidianos de la formación del Estado (pp. 213-226). Era.

Strom Života. (201l, 5 de julio). Zižek-Year of Distraction [video]. Youtube. https://www.youtube.com/ watch? $\mathrm{v}=\mathrm{ChWXYNxUFdc}$

Turner, V., y Bruner, E. (1986). The Anthropology of Experience. University of Illinois Press.

Williams, R. (2000). Marxismo y literatura. Península. 